\title{
CXCL12 chemokine genotypes as predictive biomarkers of ovarian cancer outcome
}

\author{
ANA COELHO $^{1}$, DEOLINDA PEREIRA ${ }^{2}$, ANA NOGAL ${ }^{1}$, DANIELA PINTO ${ }^{1,}$ \\ RAQUEL CATARINO $^{1}$, ANTÓNIO ARAÚJO ${ }^{2}$ and RUI MEDEIROS ${ }^{1}$ \\ ${ }^{1}$ Molecular Oncology and Virology, ${ }^{2}$ Medical Oncology, Instituto Português de Oncologia de \\ Francisco Gentil, R. Dr António Bernardino de Almeida, 4200-072 Porto, Portugal
}

Received July 15, 2008; Accepted October 29, 2008

DOI: $10.3892 / \mathrm{mmr} 00000069$

\begin{abstract}
Ovarian cancer is an aggressive disease with high mortality. The CXCL12 chemokine has been associated with the development of this neoplasia. The aim of this study was to evaluate the genetic influence of the CXCL12-3' $A$ polymorphism as a prognostic/predictive factor in ovarian cancer patients treated with platinum/paclitaxel chemotherapy. The mean survival rates for early stages (I/II) of the disease were statistically different according to patient genotype (96 months for GG and 57 months for A carrier genotypes; $\mathrm{p}=0.017$ ). The mean progression-free interval was statistically lower in patients with early stages (I/II) of the tumour carrying the A allele (55 months) than in those carrying the GG genotype (91 months; $\mathrm{P}=0.009$ ). The $C X C L 12-3$ ' $A$ polymorphism leads to a poorer response to chemotherapy with cisplatin/paclitaxel, and diminishes the mean survival rate and the progression-free interval in patients with ovarian cancer. $C X C L 12-3$ 'A may therefore serve as an important predictive biomarker for the determination of outcome in ovarian cancer.
\end{abstract}

\section{Introduction}

Ovarian cancer is the sixth most common type of cancer and ranks seventh as the most frequent cause of cancer-related death in women ( $4.0 \%$ of cases and $4.2 \%$ deaths) (1). At diagnosis, the majority of ovarian cancers have already progressed to stage III or IV, which results in poor long-term patient survival and quality of life (2). Standard treatment involves cytoreductive surgery followed by systemic platinumbased chemotherapy (3). Paclitaxel combinations with either cisplatin or carboplatin are first-line chemotherapy regimens,

Correspondence to: Ana Coelho, Instituto Português de Oncologia de Francisco Gentil, Laboratórios - Piso 4, R. Dr António Bernardino de Almeida, 4200-072 Porto, Portugal

E-mail: pequenocoelho@hotmail.com

Key words: ovarian cancer, CXCL12 chemokine, polymorphism, chemotherapy, pharmacogenomic profile each combination producing similar responses (4). This therapy results in variable efficacy rates, with frequent recurrences at even early stages of the disease, and with most tumours eventually becoming resistant to chemotherapy (5).

The biological mechanisms responsible for chemotherapy resistance remain unclear, but it is now widely accepted that the apoptotic capacity of cancer cells is pivotal in determining response to chemotherapeutic targets (6). Phosphatidylinositol 3-kinase (PI3K)/Akt (serine/threonine kinase subfamily) is a major cell survival pathway, and several targets of Akt, such as proapoptotic protein BAD and caspase-9, have roles in the regulation of apoptosis; phosphorylation by Akt inhibits their function (7).

Akt overexpression or activation is correlated with poor prognosis in several tumour types, including gastric and hepatocellular carcinoma, breast and pancreatic cancer and melanoma (8). Previous studies have proposed that PI3K/Akt activation in ovarian cancer cells promotes resistance to cisplatin-induced apoptosis, serving as a mediator in chemoresistance $(6,7)$. This activation may occur due to a variety of stimuli in different types of tumours. It has been suggested that one such stimulus is the chemokine CXCL12 (9-12).

The chemokine receptor CXCR4 and its sole ligand, SDF-1/CXCL12 (stromal cell-derived factor-1), play important roles in inflammation and hematopoiesis by acting as chemoattractants for leukocytes and stem cells (13). Furthermore, it has been demonstrated that in several types of cancer, including melanoma, ovarian, breast and lung cancer, CXCL12 can stimulate the proliferation and/or survival of CXCR4-expressing cancer cells when they are grown under suboptimal conditions (14). A growing number of studies associate CXCL12 and its receptor, CXCR4, with the development of ovarian cancer $(9,10,15,16)$.

Genetic polymorphisms may also influence outcome in cancer patients after treatment $(5,17,18)$, and a single nucleotide polymorphism in the $3^{\prime}$ untranslated region of the CXCL12 gene, a CXCL12-3'A polymorphism consisting of a $\mathrm{G} \rightarrow \mathrm{A}$ transition, was reported to be associated with susceptibility to lung and breast cancer development (19-21).

The aim of this study was to evaluate the genetic influence of the CXCL12-3'A polymorphism in epithelial ovarian cancer patients treated with platinum- and paclitaxelbased chemotherapy in order to evaluate its role as a prognostic/predictive factor in this neoplasia. 


\section{Patients and methods}

Study population. This study was conducted in 129 consecutive patients with histologically confirmed epithelial ovarian cancer (mean age 53 years) admitted to the Portuguese Institute of Oncology, Porto, Portugal, and treated with platinum-based chemotherapy between 1996 and 2002. The first-line chemotherapeutic protocol consisted of paclitaxel $\left(175 \mathrm{mg} / \mathrm{m}^{2}\right)$ and cisplatin $\left(75 \mathrm{mg} / \mathrm{m}^{2}\right)$. Patients were evaluated according to the staging system of the International Federation of Gynaecology and Obstetrics (FIGO), and the assessment of tumour response to chemotherapy was based on World Health Organization (WHO) criteria. Resistance to this treatment was determined if the disease persisted or recurred within six months after the conclusion of the chemotherapy. Patients then underwent second-line chemotherapy according to their performance status and age. If a recurrence appeared after six months of treatment, the patient underwent another course of the same combination chemotherapy (platinum/paclitaxel). All patients were treated by the same medical oncologist.

Samples were obtained with the written informed consent of the participants prior to their inclusion in the study according to the Declaration of Helsinki.

Samples. Venous blood $(\sim 8 \mathrm{ml})$ was obtained by a standard venipuncture technique using EDTA-containing tubes. DNA was extracted from the white blood cell fraction of each study subject using a salting out protocol as described by Miller et al (22).

The CXCL12-3'A genotypes were analysed using PCR at an annealing temperature of $58^{\circ} \mathrm{C}$, followed by RFLP techniques as previously described (19). The species were examined for the presence of amplifiable DNA. In each PCR experiment, two negative controls were employed to make sure that no contaminants were introduced in the initial PCR.

The 302-bp PCR products were digested with the MspI restriction enzyme (Fermentas) at $37^{\circ} \mathrm{C}$ for $1.30 \mathrm{~min}$. Restriction fragments were then visualised by agarose gel electrophoresis $(3 \%)$ with ethidium bromide staining showing three types of band patterns: wild-type homozygote $(\mathrm{G} / \mathrm{G})$, two bands corresponding to 201 and $101 \mathrm{bp}$; heterozygote (G/A), three bands corresponding to 302, 201 and $101 \mathrm{bp}$; homozygote (A/A), only one band of $302 \mathrm{bp}$. In cases of ambiguity regarding the discrimination between partial digests and apparent heterozygotes, genotyping was repeated twice using higher concentrations of the restriction enzyme to rule out partial digestion.

Statistical analysis. Analysis of data was performed using the Statistical Package for Social Sciences (SPSS) computer software for Windows (version 12.0; SPSS, Chicago, IL, USA). Differences in proportions were evaluated by the $\chi^{2}$ test or Fisher's exact test. Quantitative data were analysed using the Mann-Whitney U-test. A 5\% level of significance was applied.

The probabilities of survival and progression-free survival were calculated, and medians and life-tables were computed using the Kaplan-Meier product-limit estimate. Curves were examined by the log-rank test, a statistical test for the equality of survival distributions.

Survival duration was defined as the time between diagnosis and death or the last clinical evaluation of the patient.
Table I. Relation between CXCL12-3'A genotypic frequencies and clinicopathological parameters in ovarian cancer patients.

\begin{tabular}{lccccc}
\hline Parameter & \multicolumn{2}{c}{ GG } & \multicolumn{2}{c}{ A carrier } & \multirow{2}{*}{ P-value } \\
& No. $\%$ & No. $\%$ & \\
\hline Mean age (years) & $53.0 \pm 14.5$ & $53.6 \pm 13.5$ & $0.615^{\mathrm{a}}$ \\
FIGO stage & & & & & \\
$\quad$ I/II & 35 & 85.4 & 6 & 14.6 & $0.008^{\mathrm{b}}$ \\
III/IV & 43 & 61.4 & 27 & 38.6 & \\
Histological type & & & & & \\
$\quad$ Serous & 37 & 66.1 & 19 & 33.9 & $0.277^{\mathrm{b}}$ \\
$\quad$ Other types & 32 & 76.2 & 10 & 23.8 & \\
\hline
\end{tabular}

${ }^{\mathrm{a}}$ Mann-Whitney U-test;. ${ }^{\mathrm{b}} \chi^{2}$ test.

Cause of death was determined according to patient records, death certificates or communication with the general practitioner handling the case. Progression-free interval survival was defined as the time interval between diagnosis and the occurrence of an event (death, recurrence, progression of disease) or the last clinical evaluation.

The observed number of each genotype was compared with that expected for a population in Hardy-Weinberg equilibrium.

\section{Results}

The analysis of CXCL12-3'A genotypes in women with ovarian cancer was only possible in 111 of the initial 129 women enrolled in this study due to DNA extraction or amplification problems. This group of cases was divided into subgroups according to clinical stages I/II (early stages) and III/IV (advanced stages). The genotypic frequencies observed were $70.3 \%$ for $\mathrm{GG}, 25.2$ for $\mathrm{AG}$ and $4.5 \%$ for $\mathrm{AA}$, and did not differ from those expected from the Hardy-Weinberg equilibrium $(\mathrm{P}=0.71)$.

Table I shows the relation between CXCL12-3'A genotypic frequencies and clinicopathological parameters. There were no statistically significant differences between the groups of patients with the different genotypes (GG and A carriers) regarding age at diagnosis and the histologic type of ovarian cancer. There was a statistically significant overrepresentation of A carrier genotypes in the clinical stage group III/IV of ovarian cancer in comparison with the less aggressive stages $\mathrm{I} / \mathrm{II}(\mathrm{P}=0.008)$.

We used Kaplan-Meier methodology to analyse overall survival rates (Fig. 1). The mean survival rates for stages I/II were statistically different according to patient genotype (96 months for GG and 57 months for A carrier genotypes; $\mathrm{p}=0.017)$. Regarding stages III/IV, there was no statistical difference (56 months for GG and 62 months for A carrier genotypes; $\mathrm{P}=0.834$ ).

Concerning the progression-free interval (Fig. 2), we observed that the mean progression-free interval was statistically lower in patients carrying the A allele with early stages (I/II) of the tumour (55 months) than in those carrying the GG genotype (91 months; $\mathrm{P}=0.009$ ). In advanced stages of the 
A

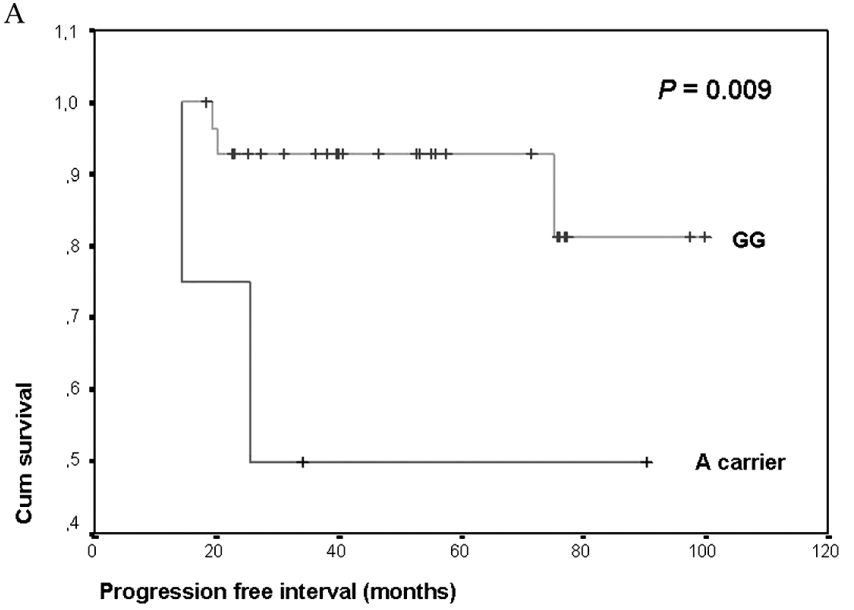

B

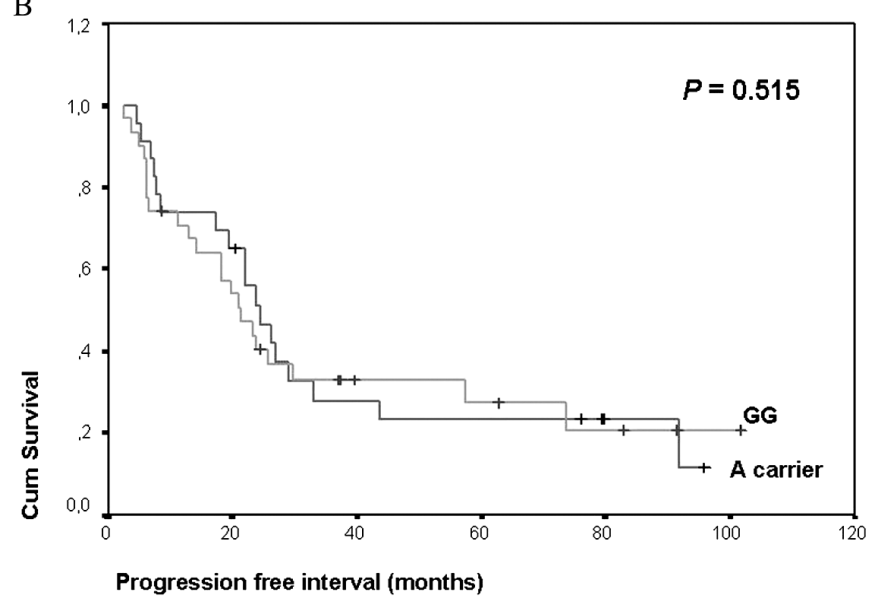

Figure 1. Association of CXCL12-3'A genotypes with overall survival by Kaplan-Meier curves. Comparison between GG and A carrier genotypes in (A) early (I/II) and (B) advanced (III/IV) stages of ovarian cancer.

A

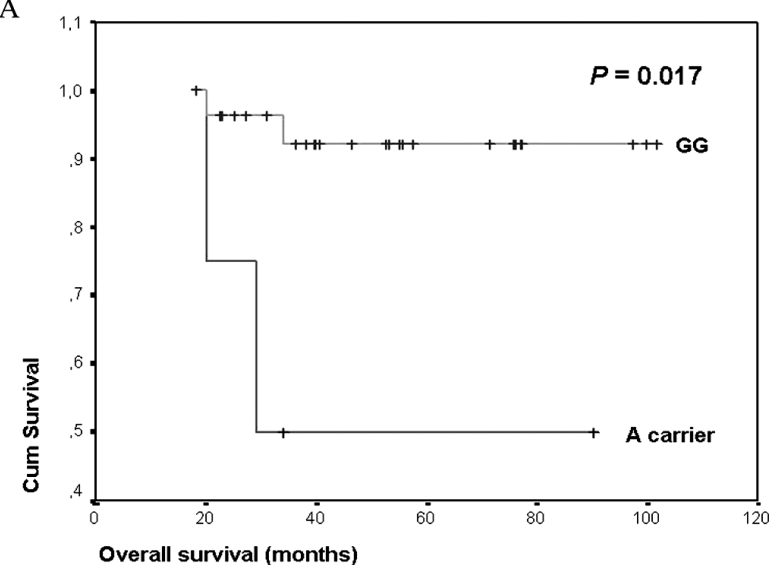

B

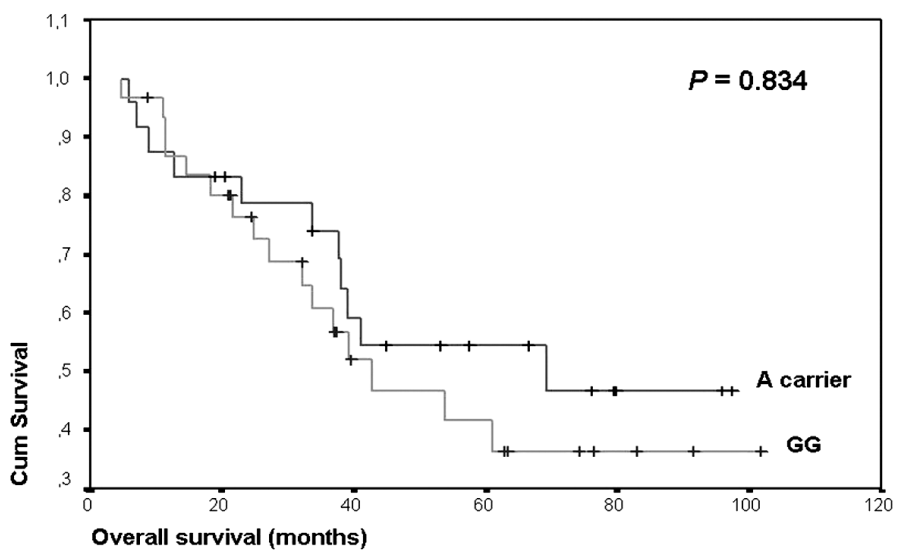

Figure 2. Association of CXCL12-3'A genotypes with progression-free interval by Kaplan-Meier curves. (A) Comparison between GG and A carrier genotypes in (A) early (I/II) and (B) advanced (III/IV) stages of ovarian cancer.

disease (III/IV), no statistical difference was observed regarding the mean progression-free interval in patients presenting the GG genotype (39 months) and in those presenting the genotypes carrying the A allele ( 37 months; $\mathrm{P}=0.515$ ).

\section{Discussion}

Ovarian cancer is an aggressive disease with high mortality due in large part to high rates of therapy failure and resistance and to consequent recurrences. It is now accepted that chemotherapy for ovarian cancer is limited by significant interindividual variations, which are often associated with genetic variations (polymorphisms) in specific genes $(23,24)$.

The present findings support the hypothesis that the CXCL12-3'A polymorphism has a strong influence on the outcome of ovarian cancer in patients treated with cisplatin/ paclitaxel chemotherapy, indicating that mean survival rates and the progression-free interval are influenced by CXCL123'A genotype subgroups (A carrier and GG) in early stages (I/II) of the disease $(\mathrm{P}=0.017$ and 0.009 , respectively). The A carrier genotypes are associated with a poorer prognosis, conferring lower overall survival and progression-free interval when compared with the GG genotype independently of age and histologic tumour type. No statistically significant differences were found regarding the influence of these genotypes on advanced stages (III/IV) of ovarian cancer.

Our results are in accordance with several reports suggesting that strong differences exist between early and advanced ovarian cancer in terms of overall survival, treatment response and tumour behaviour (25-29). In addition, we found a difference between A carrier frequencies in early and advanced stages of the disease $(\mathrm{P}=0.008)$, suggesting that stage I/II and III/IV ovarian tumours have distinct cell populations.

The assumption that tumours can contain drug-sensitive, physiologically drug-resistant and pathologically drug-resistant cells explains several paradoxical observations in the biology of ovarian cancer. Clinical response to chemotherapy is determined by the relative contribution of these different cell populations to the total cellular mass of a tumour (30).

In some types of cancer, CXCL12 can stimulate cancer cell proliferation or survival under suboptimal conditions. In ovarian cancer, there is abundant and tumour-specific expression of CXCL12 (9). The chemokine was also found in the ascitic fluid of ovarian cancer patients (16). There is also evidence to suggest that CXCL12 mediates the proliferative actions of estrogens in ovarian cancer cells (31). As a result, 
CXCL12 may provide important paracrine and autocrine signals that promote malignant progression in ovarian cancer (9).

Stimulation of the chemokine receptor CXCR 4 by its ligand, CXCL12, results in the activation of the ERK-2 and PI3K signalling pathways, leading to the prolonged activation of PKB/Akt $(12,32)$. PI3K/Akt signalling pathways are frequently disturbed in many human cancers, and play a major role not only in tumour development, but also in the potential response of tumours to cancer treatment (33). In ovarian cancer, aberrant Akt expression or activation in different cell lines has been found to confer paclitaxel (33) and cisplatin $(6,7)$ resistance, serving as a mediator of chemoresistance.

CXCL12-3'A seems to be a functional polymorphism, altering the expression levels of the chemokine CXCL12 $(20,34)$. It is our belief that the presence of the polymorphism (through the overexpression of CXCL12) contributes to the prolonged activation of Akt in ovarian cancer cells, thus leading to a poorer response to combination chemotherapy with cisplatin/paclitaxel and diminishing the mean survival rates and the progression-free interval in patients with ovarian cancer cells. This is consistent with our results.

The CXCL12-3'A polymorphism is an important predictive biomarker in a panel of genetic factors conditioning the outcome of ovarian cancer and delineating the biologic mechanisms responsible for induced drug resistance. Identification of novel therapies designed to minimise this effect should be the focus of further research.

\section{Acknowledgements}

The authors would like to thank the Liga Portuguesa Contra o Cancro for their support. This study was supported by a fund from the Comissão de Fomento à Investigação em Cuidados de Saúde, Ministério da Saúde P.I. no. 31/2007.

\section{References}

1. Parkin DM, Bray F, Ferlay J and Pisani P: Global Cancer Statistics, 2002. CA Cancer J Clin 55: 74-108, 2005.

2. Gupta SK, John S, Naik R, et al: A multicenter phase II study of gemcitabine, paclitaxel, and cisplatin in chemonaive advanced ovarian cancer. Gynecol Oncol 98: 134-140, 2005.

3. Mutch DG: Surgical management of ovarian cancer. Semin Oncol 29: 3-8, 2002

4. Ozols RF, Bundy BN, Greer BE, et al: Phase III trial of carboplatin and paclitaxel compared with cisplatin and paclitaxel in patients with optimally resected stage III ovarian cancer: a Gynecologic Oncology Group study. J Clin Oncol 21: 3194-3200, 2003.

5. Pinto D, Pereira D, Portela C, Da Silva JL, Lopes C and Medeiros R: The influence of HER2 genotypes as molecular markers in ovarian cancer outcome. Biochem Biophys Res Commun 335: 1173-1178, 2005.

6. Fraser M, Leung BM, Yan X, Dan HC, Cheng JQ and Tsang BK: p53 is a determinant of X-linked inhibitor of apoptosis protein/ Akt-mediated chemoresistance in human ovarian cancer cells. Cancer Res 63: 7081-7088, 2003.

7. Yuan ZQ, Feldman RI, Sussman GE, Coppola D, Nicosia SV and Cheng JQ: AKT2 inhibition of cisplatin-induced JNK/p38 and Bax activation by phosphorylation of ASK1: implication of AKT2 in chemoresistance. J Biol Chem 278: 23432-23440, 2003.

8. Dai DL, Martinka M and Li G: Prognostic significance of activated Akt expression in melanoma: a clinicopathologic study of 292 cases. J Clin Oncol 23: 1473-1482, 2005.

9. Scotton CJ, Wilson JL, Scott K, et al: Multiple actions of the chemokine CXCL12 on epithelial tumor cells in human ovarian cancer. Cancer Res 62: 5930-5938, 2002.
10. Porcile C, Bajetto A, Barbieri F, et al: Stromal cell-derived factor-1alpha (SDF-1alpha/CXCL12) stimulates ovarian cancer cell growth through the EGF receptor transactivation. Exp Cell Res 308: 241-253, 2005.

11. Eaves CJ: SDF-1 tells stem cells to mind their P's and Z's. J Clin Invest 115: 27-29, 2005.

12. Barbero S, Bonavia R, Bajetto A, et al: Stromal cell-derived factor 1alpha stimulates human glioblastoma cell growth through the activation of both extracellular signal-regulated kinases $1 / 2$ and Akt. Cancer Res 63: 1969-1974, 2003.

13. Samara GJ, Lawrence DM, Chiarelli CJ, et al: CXCR4-mediated adhesion and MMP-9 secretion in head and neck squamous cell carcinoma. Cancer Lett 214: 231-241, 2004.

14. Balkwill F: Cancer and the chemokine network. Nat Rev Cancer 4: 540-550, 2004.

15. Kryczek I, Lange A, Mottram P, et al: CXCL12 and vascular endothelial growth factor synergistically induce neoangiogenesis in human ovarian cancers. Cancer Res 65: 465-472, 2005.

16. Scotton CJ, Wilson JL, Milliken D, Stamp G and Balkwill FR: Epithelial cancer cell migration: a role for chemokine receptors? Cancer Res 61: 4961-4965, 2001

17. Medeiros R, Pereira D, Afonso N, et al: Platinum/paclitaxelbased chemotherapy in advanced ovarian carcinoma: glutathione S-transferase genetic polymorphisms as predictive biomarkers of disease outcome. Int J Clin Oncol 8: 156-161, 2003.

18. Modugno F: Ovarian cancer and polymorphisms in the androgen and progesterone receptor genes: a HuGE review. Am J Epidemiol 159: 319-335, 2004.

19. Coelho A, Calcada C, Catarino R, Pinto D, Fonseca G and Medeiros R: CXCL12-3'A polymorphism and lung cancer metastases protection: new perspectives in immunotherapy? Cancer Immunol Immunother 55: 639-643, 2005.

20. Razmkhah M, Talei AR, Doroudchi M, Khalili-Azad T and Ghaderi A: Stromal cell-derived factor-1 (SDF-1) alleles and susceptibility to breast carcinoma. Cancer Lett 225: 261-266, 2005.

21. Zafiropoulos A, Crikas N, Passam AM and Spandidos DA: Significant involvement of CCR2-64I and CXCL12-3a in the development of sporadic breast cancer. J Med Genet 41: e59, 2004.

22. Miller SA, Dykes DD and Polesky HF: A simple salting out procedure for extracting DNA from human nucleated cells. Nucleic Acids Res 16: 1215, 1988.

23. Abuharbeid S, Apel J, Sander M, et al: Cytotoxicity of the novel anti-cancer drug rViscumin depends on HER-2 levels in SKOV-3 cells. Biochem Biophys Res Commun 321: 403-412, 2004.

24. Chen X, Yeung TK and Wang Z: Enhanced drug resistance in cells coexpressing ErbB2 with EGF receptor or ErbB3. Biochem Biophys Res Commun 277: 757-763, 2000.

25. Friedlander ML, Taylor IW, Russell P, Musgrove EA, Hedley DH and Tattersall MH: Ploidy as a prognostic factor in ovarian cancer. Int J Gynecol Pathol 2: 55-63, 1983.

26. Roberts PC, Mottillo EP, Baxa AC, et al: Sequential molecular and cellular events during neoplastic progression: a mouse syngeneic ovarian cancer model. Neoplasia 7: 944-956, 2005.

27. Saretzki G, Hoffmann U, Rohlke P, et al: Identification of allelic losses in benign, borderline, and invasive epithelial ovarian tumors and correlation with clinical outcome. Cancer 80: 1241-1249, 1997.

28. Shridhar V, Lee J, Pandita A, et al: Genetic analysis of earlyversus late-stage ovarian tumors. Cancer Res 61: 5895-5904, 2001.

29. Smith DI: Transcriptional profiling develops molecular signatures for ovarian tumors. Cytometry 47: 60-62, 2002.

30. Pusztai L, Siddik ZH, Mills GB and Bast RC Jr: Physiologic and pathologic drug resistance in ovarian carcinoma - a hypothesis based on a clonal progression model. Acta Oncol 37: 629-640, 1998.

31. Hall JM and Korach KS: Stromal cell-derived factor 1, a novel target of estrogen receptor action, mediates the mitogenic effects of estradiol in ovarian and breast cancer cells. Mol Endocrinol 17: 792-803, 2003.

32. Balkwill F: The significance of cancer cell expression of the chemokine receptor CXCR4. Semin Cancer Biol 14: 171-179, 2004.

33. Fresno Vara JA, Casado E, De Castro J, Cejas P, Belda-Iniesta C and Gonzalez-Baron M: PI3K/Akt signalling pathway and cancer. Cancer Treat Rev 30: 193-204, 2004.

34. Soriano A, Martinez C, Garcia F, et al: Plasma stromal cellderived factor (SDF)-1 levels, SDF1-3'A genotype, and expression of CXCR4 on T lymphocytes: their impact on resistance to human immunodeficiency virus type 1 infection and its progression. J Infect Dis 186: 922-931, 2002. 